Recepción: 15 / 04 / 2017

Ciencias Administrativas

Aceptación: 01 / 05 / 2017

Articulo Científico

Publicación: 15 / 05 / 2017

\title{
Aporte del biocomercio como fuente de fomento y desarrollo sostenible
}

\section{Contribution of trade as a source of development and sustainable development}

\section{Contribuição do comércio como fonte de promoção e desenvolvimento sustentável}

Silvia A. Medina-Anchundia silvia.medinaa@ug.edu.ec

Juan C. Ramos-Romero"

juan.ramosr@ug.edu.ec

Janet E. Pazmiño-Ramirez ${ }^{\text {"I }}$ janet.pazmiñor@ug.edu.ec

Correspondencia: silvia.medinaa@ug.edu.ec

Magister en Administración de Empresas; Ingeniera Comercial, Universidad de Guayaquil, Ecuador.

Magister en Docencia y Gerencia en Educación Superior; Ingeniero en Sistemas Computacionales; Universidad de Guayaquil, Ecuador.

III. Magister en Diseño Curricular; Profesora Especialización Educación Primaria; Licenciada en Ciencias de la Educación Especialización Educación Primaria; Profesora de Segunda Enseñanza en la Especialización de Comercio y Administración; Universidad de Guayaquil, Ecuador. 


\section{Resumen}

La Iniciativa del Biocomercio en el Ecuador (IB), apareció por primera vez en la Conferencia de las Naciones Unidas sobre Comercio y Desarrollo - UNCTAD, durante la tercera Conferencia de las Partes (COP III) del Convenio de la Diversidad Biológica (CDB), la cual se llevó a cabo en noviembre de 1996 en Argentina. Con el objetivo de incitar el comercio y las inversiones en recursos biológicos y promover el desarrollo sostenible, sus principios fundamentales son: Preservación de la diversidad biológica, uso sustentable de sus componentes y participación justa y ecuánime de los beneficios que se derivan de la utilización de los recursos biológicos y genéticos. El Biocomercio busca mejorar las capacidades de los países en desarrollo, para usar en forma sostenible la biodiversidad. Esta investigación se ha basado principalmente en recopilar y analizar la información del sector, de los habitantes y las actividades a las que se dedican en el Recinto Piscano del Cantón Palenque de la Provincia de Los Ríos; actividades que están relacionadas con la agricultura, flora y fauna, y a la producción agroindustrial, información que se analizó de forma cuantitativa y cualitativa a través de diferentes métodos y técnicas como es la observación directa por visita in situ con sus respectivos instrumentos, utilizando a la encuesta como técnica de investigación la cual fue aplicada a todas las personas mayores que forman parte del Recinto Piscano. Los resultados obtenidos nos permiten generar propuestas para activar a los sectores productivos .Con esta investigación determinamos que el biocomercio puede ser fomentado, aplicado y desarrollado en las comunidades de Palenque, mediante la aplicación de modelos que nos permitan generar proyectos de innovación para el mejoramiento económico de la población.

Palabras clave: Biocomercio; industria alimenticia; industria farmacéutica y cosmética; turismo sostenible. 


\begin{abstract}
The BIOTRADE initiative in Ecuador (IB), first appeared in the United Nations Conference on trade and Development - UNCTAD, during the third Conference of the parties (COP III) of the Convention of the biological diversity (CBD), which was held in November 1996 in Argentina. With the objective of encourage the trade and them investments in resources biological and promote the development sustainable, their principles fundamental are: preservation of the diversity biological, use sustainable of their components and participation fair and fair of them benefits that is derived of the use of them resources biological and genetic. BIOTRADE aims to improve the capacities of developing countries, to use biodiversity in a sustainable manner. This research has been based mainly on collecting and analyzing the information sector, the inhabitants and activities that engage in the enclosure Piscano of the Canton Palenque of the province's rivers; activities that are related with the agriculture, flora and fauna, and to the production agro-industrial, information that is analyzed of form quantitative and qualitative through different methods and technical as is the observation direct by visit on-site with their respective instruments, using to the survey as technical of research which was applied to all them people older that form part of the enclosure Piscano. The results obtained us allow generate proposals to activate to the sectors productive. With this research determined that the BIOTRADE can be fostered, applied and developed in the communities of Palenque, through the application of models that us allow generate projects of innovation for the improvement economic of the population.
\end{abstract}

Key words: Biotrade; food industry; pharmaceuticals and cosmetics; sustainable tourism. 


\section{Resumo}

A iniciativa BIOTRADE no Equador (IB), surgiu pela primeira vez na Conferência das Nações Unidas sobre Comércio e Desenvolvimento - UNCTAD, durante a terceira Conferência das Partes (COP III) da Convenção sobre Diversidade Biológica (CBD), realizada em novembro 1996 na Argentina. Com o objetivo de incentivar o comércio e os investimentos em recursos biológicos e promover o desenvolvimento sustentável, seus princípios fundamentais são: preservação da diversidade biológica, uso sustentável de seus componentes e participação justa e justa dos benefícios derivados do uso de Recursos biológicos e genéticos. A BIOTRADE visa melhorar as capacidades dos países em desenvolvimento, utilizar a biodiversidade de forma sustentável. Esta pesquisa tem sido baseada principalmente na coleta e análise do setor de informação, os habitantes e atividades que envolvem no recinto Piscano do Cantão Palenque dos rios da província; Atividades que se relacionam com a agricultura, flora e fauna, e à produção agroindustrial, informação que é analisada de forma quantitativa e qualitativa através de diferentes métodos e técnicas como é a observação direta por visita no local com seus respectivos instrumentos, utilizando À pesquisa como técnica de pesquisa que se aplicou a todos os povos mais velhos que fazem parte do cerco Piscano. Os resultados obtidos permitem gerar propostas para ativar aos setores produtivos. Com esta pesquisa determinou-se que a BIOTRADE pode ser fomentada, aplicada e desenvolvida nas comunidades de Palenque, através da aplicação de modelos que permitam gerar projetos de inovação para a melhoria econômica da população.

Palavras chave: Biotrade; indústria alimentícia; produtos farmacêuticos e cosméticos; turismo sustentável. 


\section{Introducción.}

El cantón Palenque de la Provincia de Los Ríos, está ubicado geográficamente en el centro del País, zona 5 según lo establece el modelo de división política y comercial de la Secretaria Nacional de Planificación y Desarrollo (SENPLADES); es un sector donde la práctica del biocomercio tendría una gran acogida, debido a las riquezas de su tierra en la cual existe una gran variedad de biodiversidad.

Debido a la crisis económica global, el biocomercio sería una gran fuente de ingreso y desarrollo en sus comunidades, la ONU en el año 2007 en la Conferencia de las Naciones Unidas sobre Comercio y Desarrollo precisa que el Biocomercio son aquellas actividades de producción, recolección, transformación y comercialización de bienes y servicios derivados de la biodiversidad nativa (recursos genéticos, especies y ecosistemas) que involucran prácticas de conservación y uso sostenible, y son generados con criterios de sostenibilidad ambiental, social y económica. (Guzman L \& Gonzalez A, 2004) (Instituto de investigación de recursos biológicos Alexander Von Humboldt, 2000)

La importancia de aprovechar de mejor manera los ecosistemas reside en la conservación de los recursos naturales, de allí parte la idea de generar productos y servicio sostenible que logre mejorar el aspecto social y ambiental de la comunidad y las especies que sobre ellos habitan. (Salvador \& Gómez-Sal, 2003) (Scheffer, Carpenter, Foley, Folke, \& Walke, 2001) (Miller, 1990)

El presente proyecto tiene como finalidad identificar las actividades económicas del biocomercio en el cantón Palenque, específicamente en la zona rural donde se desarrolla el sector agrícola; lo cual permitirá determinar el fomento del desarrollo sostenible en las comunidades del cantón. 


\section{Materiales y métodos.}

La investigación tiene una perspectiva profesional, se ha fundamentado primordialmente en recopilar y examinar la información del sector, de los habitantes y las actividades a las que se dedican en el Recinto Piscano del Cantón Palenque de la Provincia de Los Ríos; actividades que estarían vinculadas con la agricultura, flora y fauna, y la producción agroindustrial, información que se examinará de forma cuantitativa y cualitativa a través de diferentes métodos y técnicas como es la observación directa por visita in situ con sus pertinentes instrumentos y los resultados serán evaluados que permitan generar propuestas para activar a los sectores productivos basados en la agenda zonal 5 y la matriz productiva.

Con esta investigación se podrá determinar si el biocomercio se puede fomentar, aplicar y desarrollar en las comunidades de Palenque, mediante la aplicación de modelos que permita generar proyectos de innovación para el mejoramiento económico de la población.

\section{Modalidad y tipo de investigación}

La particularidad a utilizar para la preparación del trabajo, es la investigación cualitativacuantitativa, donde lo cualitativo hace énfasis en solucionar el problema que se genera por el desconocimiento, la deforestación y la contaminación de la tierra por la carencia de conocimientos sobre el biocomercio y el paradigma cuantitativo se describe a los datos generados de la encuesta.

Cabe señalar que se puntualizan las propiedades que se analizarán, también se miden y se evalúan las dimensiones del estudio de la asociatividad, tales como el mecanismo de cooperación, procesos colectivos, trabajo en equipo, unificación empresarial, uso de herramientas, comercialización, propósitos de asociarse, ventajas competitivas, conformación de una asociación, 
nivel de tecnología empleado, se evaluará cada una de ellas de forma autónoma, para así proceder a instaurar lo que se indaga.

A través de esta particularidad resalto la concordancia que existe entre las variables modelo asociativo y los cultivadores, dicha indagación busca expresar las cualidades importantes del grupo de agricultores y ganaderos del recinto Piscano del cantón Palenque de la Provincia de Los Ríos, para ser sujeto a análisis. Es trascendental indicar que a través de esta indagación se evalúan de modo independiente las variables e integran las mediciones de cada una de las indicadas variables con el objeto de que se presente el fenómeno de interés, su objetivo no es mostrar cómo se dependen las variables medidas, sino puntualizar cada una de ellas.

La población es "un conjunto finito o infinito de elementos con características comunes para los cuales serán extensivas las conclusiones de la investigación”.

Para el estudio del biocomercio se tomará como población los habitantes del recinto Piscano del canto Palenque de la provincia de Los Ríos, va dirigido a 105 personas conformadas por 35 familias y que todos se dedican a la agricultura. De acuerdo a datos proporcionados por el (GAP, 2015), de esta población es importante señalar cuales serían las estrategias que ellos adoptan para referencia de la propuesta planteada en el presente estudio.

Para la actual investigación se efectuará la estadística descriptiva; la cual recolecta, presenta y caracteriza un conjunto de datos con la finalidad de detallar aproximadamente las características de este. 
El investigador, tiene como finalidad detallar situaciones y sucesos de esta manera definir las propiedades importantes de las personas, grupos, comunidades o cualquier otro fenómeno que sea sometido a análisis.

Mediante la estadística descriptiva se recopilará la indagación, procesando los datos al organizarlos en frecuencias y porcentajes.

\section{Resultados.}

Se aplicó esta encuesta a 105 personas adultas que conforman las 35 familias del Recinto Piscano del cantón Palenque provincia de Los Ríos.

Este bloque de preguntas me permitió adquirir información socioeconómica tales como la educación, salud, educación sexual, servicios básicos.

\section{Pregunta $N^{\circ}$ 1: Edad}

$\mathrm{Al}$ realizar la encuesta se evidencia que las personas del recinto tienen edades comprendidas entre 25 a 30 años que representan un $43 \%$ de la población, entre 31 a 40 años tiene un 34\%, entre 41 a 50 años tiene un $17 \%$ y mayores o iguales a 51 años tienen un 6\%. Esto da como conclusión que la mayor parte de la población son personas con edad para generar emprendimiento.

\begin{tabular}{ccc} 
DATOS DE EDADES & CANTIDAD & $\%$ \\
\hline $25-30$ & 45 & $43 \%$ \\
$31-40$ & 36 & $34 \%$ \\
$41-50$ & 18 & $17 \%$ \\
$>=51$ & 6 & $6 \%$ \\
TOTAL & $\mathbf{1 0 5}$ & $\mathbf{1 0 0 \%}$
\end{tabular}

Tabla $N^{\circ}$ 1.- Edad del encuestado 


\section{Pregunta $N^{\circ}$ 2: Sexo}

Podemos indicar que los datos promedio del sexo en la encuesta realizada nos muestran como resultado que el sexo masculino tiene un porcentaje del $56 \%$, en cuanto al sexo femenino tuvo un porcentaje del $44 \%$. Con esto nos podemos dar cuenta que el mayor porcentaje de la población es del sexo masculino. Como conclusión podemos manifestar que tanto el sexo masculino como el femenino pueden trabajar en conjunto para implementar al biocomercio en el recinto Piscano.

\begin{tabular}{ccc} 
DATOS DEL SEXO & CANTIDAD & \% \\
\hline Masculino & 59 & $56 \%$ \\
Femenino & 46 & $44 \%$ \\
TOTAL & 105 & $100 \%$
\end{tabular}

Tabla $N^{\circ}$ 2.- Sexo del encuestado

\section{Pregunta $N^{\circ}$ 3: Estado Civil}

Los datos promedio del estado civil realizados en la encuesta nos dieron como resultado, que existe un porcentaje del $58 \%$ de personas casadas, un $14 \%$ es representado por las personas viudas y las personas divorciadas están representadas por un $28 \%$. En este caso las personas casadas cuentan con un mayor índice de porcentaje en la población.

\begin{tabular}{ccc}
$\begin{array}{c}\text { DATOS DE ES TADO } \\
\text { CIVIL }\end{array}$ & CANTIDAD & $\%$ \\
\hline Casado & 61 & $58 \%$ \\
Viudo & 15 & $14 \%$ \\
Divorciado & 29 & $28 \%$ \\
TOTAL & 105 & $100 \%$
\end{tabular}

Tabla $N^{\circ}$ 3.- Estado civil de encuestado 


\section{Pregunta $N^{\circ}$ 4: Actividad}

La encuesta nos muestra los datos promedios de las actividades a las que se dedican las personas del recinto Piscano, obteniendo un porcentaje del 68\%en la agricultura, la ganadería con un $21 \%$ y en el turismo un $11 \%$. Como nos indican los resultados podemos observar que la mayoría de las personas se dedican a la agricultura, seguida de la ganadería y con un menor porcentaje en el turismo. Como conclusión podemos indicar que se debe explotar los tres aspectos los cuales son fundamentales para el recinto.

\begin{tabular}{ccc} 
DATOS DE ACTIVIDAD & CANTIDAD & \% \\
\hline Agricultura & 71 & $68 \%$ \\
Ganaderia & 22 & $21 \%$ \\
Turismo & 12 & $11 \%$ \\
TOTAL & 105 & $100 \%$
\end{tabular}

Tabla $N^{\circ}$ 4.- Actividad del encuestado

\section{Pregunta $N^{\circ}$ 5: Instrucción}

Los datos promedios de la instrucción en la encuesta realizada nos dieron como resultado, que existe un $70 \%$ de nivel primario, nivel de secundaria con un $30 \%$ y en el nivel superior representado por el $0 \%$. Como podemos observar la mayor parte de la población solo ha realizado el nivel de instrucción primario, siendo el nivel superior el más bajo. La mayor parte está dedicada a cultivar el campo y dejan a un lado los estudios.

\begin{tabular}{ccc} 
DATOS DE & CANTIDAD & $\%$ \\
INS TRUCCIÓN & 73 & $70 \%$ \\
\hline Primario & 32 & $30 \%$ \\
Secundario & 0 & $0 \%$ \\
Superior & 105 & $100 \%$ \\
TOTAL & &
\end{tabular}

Tabla $N^{\circ}$ 5.- Instrucción del encuestado 
Resultado del bloque datos de información

Este bloque de preguntas me permitió conocer si los habitantes del sector cuentan con el conocimiento sobre el biocomercio, la producción de la zona, la flora y fauna nativa del sector. Además, permitió conocer si los habitantes estarían dispuestos a trabajar en proyectos innovadores para proteger la biodiversidad, dejando a un lado lo que producen en la actualidad a través de químicos, lo cual causa un gran daño al ecosistema por ende afecta a la salud de las personas.

\section{Pregunta $\mathbf{N}^{\circ}$ 1: ¿Conoce usted qué es biocomercio?}

La encuesta nos da a conocer que el $86 \%$ de la población no conoce que es el biocomercio, mientras que el 14\% restante si tiene conocimientos. Esto da como conclusión que la mayor parte de la población debe recibir talleres para que adquieran los conocimientos necesarios sobre lo que es el biocomercio y puedan implementarlos en proyectos innovadores para poder contribuir al buen vivir.

\begin{tabular}{ccc} 
RESPUESTA & CANTIDAD & \% \\
\hline SI & 15 & $14 \%$ \\
NO & 90 & $86 \%$ \\
TOTAL & 105 & $100 \%$
\end{tabular}

Tabla $N^{\circ}$ 6.- ¿Conoce usted qué es biocomercio?

Pregunta $N^{\circ}$ 2: ¿Estaría de acuerdo en participar en capacitaciones técnicas relacionadas al agro, medio ambiente, ecología y comercio?

El 57\% de la población está totalmente de acuerdo en participar en capacitaciones técnicas relacionadas al agro, medio ambiente, ecología y comercio, el 16\% está de acuerdo, un $20 \%$ no está ni de acuerdo, ni en desacuerdo, el $5 \%$ está en desacuerdo y el $2 \%$ restante está totalmente en desacuerdo. Como conclusión la gran mayoría de los habitantes si muestran un gran interés por 
recibir capacitaciones sobre el biocomercio, ya que saben que les generará nuevas fuentes de ingresos y un mejor estilo de vida, dejando a un lado todo lo que tenga que ver con químicos, los cuales solo están perjudicando su salud.

\begin{tabular}{ccc} 
RES PUES TA & CANTIDAD & \% \\
\hline Totalmente de acuerdo & 60 & $57 \%$ \\
De acuerdo & 17 & $16 \%$ \\
Ni de acuerdo, Ni en & 21 & $20 \%$ \\
desacuerdo & 5 & $5 \%$ \\
En desacuerdo & 2 & $2 \%$ \\
Totalmente en desacuerdo & 105 & $100 \%$ \\
TOTAL & &
\end{tabular}

Tabla $N^{\circ}$ 7.- ¿Estaría de acuerdo en participar en capacitaciones técnicas relacionadas al agro, medio ambiente, ecología y comercio?

Pregunta $\mathrm{N}^{\circ}$ 3: ¿Está de acuerdo que se implemente un plan de estrategias de biocomercio en el recinto?

El $70 \%$ de la población está de acuerdo en que se implemente un plan de estrategias de biocomercio en el recinto y el 30\% restante no está de acuerdo. El resultado de la encuesta nos indica según el porcentaje obtenido (70\%), las personas están interesas en progresar, en tener mayor riqueza para el bienestar de su familia.

\begin{tabular}{ccc} 
RES PUESTA & CANTIDAD & \% \\
\hline SI & 74 & $70 \%$ \\
NO & 31 & $30 \%$ \\
TOTAL & 105 & $100 \%$
\end{tabular}

Tabla $N^{\circ}$ 8.- ¿Está de acuerdo que se implemente un plan de estrategias de biocomercio en el recinto? 
Pregunta $\mathrm{N}^{\circ}$ 4: ¿Estaría dispuesto a cambiar su producción actual por nuevos cultivos que no dañe el medio ambiente o el ecosistema?

Según los resultados obtenidos el $86 \%$ de la población dio como respuesta que si están dispuestos a cambiar su producción actual por experimentar con nuevos cultivos que no dañen al medio ambiente y el $14 \%$ restante que se quedan con sus cultivos normales. Los habitantes en un gran porcentaje $(86 \%)$, están dispuestos a cambiar sus cultivos los cuales los realizan a través de fertilizantes, químicos por nuevos cultivos los cuales cuiden el medio ambiente y su salud.

\begin{tabular}{ccc} 
RES PUESTA & CANTIDAD & $\%$ \\
\hline SI & 90 & $86 \%$ \\
NO & 15 & $14 \%$ \\
TOTAL & 105 & $100 \%$
\end{tabular}

Tabla $N^{\circ}$ 9.- ¿Estaría dispuesto a cambiar su producción actual por nuevos cultivos que no dañe el medio ambiente o el ecosistema?

Pregunta $\mathrm{N}^{\circ}$ 5: ¿Le gustaría participar en proyectos que le permita a los habitantes del recinto asociarse para formar nuevas fuentes de ingresos basados en la biodiversidad, la ecología, productos orgánicos y ecoturismo?

A través de los resultados establecidos por la encueta el $86 \%$ de la población que respondió la anterior pregunta sobre si estaba dispuesto a cambiar el sistema de cultivo por uno más ecológico, así mismo respondieron que están dispuesto a participar en proyectos que les permitan a los habitantes del recinto asociarse y formar nuevas fuentes de ingresos basados en la biodiversidad, ecología, productos orgánicos y ecoturismo. La gran mayoría están dispuestos a formar asociaciones para adquirir nuevas fuentes de ingresos basados en la biodiversidad. 
Aporte del biocomercio como fuente de fomento y desarrollo sostenible

\begin{tabular}{ccc} 
RESPUESTA & CANTIDAD & \% \\
\hline SI & 90 & $86 \%$ \\
NO & 15 & $14 \%$ \\
TOTAL & 105 & $100 \%$
\end{tabular}

Tabla $N^{\circ}$ 10.- Le gustaría participar en proyectos que le permita a los habitantes del recinto asociarse para formar nuevas fuentes de ingresos basados en la biodiversidad, la ecología, productos orgánicos y ecoturismo?

\section{Pregunta $\mathbf{N}^{\circ}$ 6: ¿Desea que se mejore la productividad del sector?}

El $100 \%$ de la población encuestada nos respondió que si desean que mejore la producción del sector para así poder aumentar sus ingresos económicos.

\begin{tabular}{ccc} 
RESPUESTA & CANTIDAD & \% \\
\hline SI & 105 & $100 \%$ \\
NO & 0 & $0 \%$ \\
TOTAL & 105 & $100 \%$
\end{tabular}

Tabla $N^{\circ}$ 10.- ¿Desea que se mejore la productividad del sector?

Pregunta $\mathbf{N}^{\circ}$ 7: ¿Qué tan importante considera que sus productos sean comercializados a nivel local, provincial y nacional, mediante la producción agroindustrial?

El 89\% de la población les pareció muy importante que sus productos sean comercializados a nivel local, provincial y nacional mediante la producción agroindustrial y el $11 \%$ le pareció importante. Como conclusión esto indica que la gran mayoría de los habitantes del Reciento Piscano del cantón Palenque Provincia de los Ríos, ven en el biocomercio una nueva forma de vivir adquiriendo nuevos ingresos económicos y nuevos estilos de producir sus productos que al ser 
consumidos nos les causara ningún daño a su salud, e hicieron conciencia que debemos cuidar de nuestra biodiversidad nativa.

\begin{tabular}{ccc} 
RES PUES TA & CANTIDAD & $\%$ \\
\hline Muy importantes & 93 & $89 \%$ \\
Importantes & 12 & $11 \%$ \\
Poco importantes & 0 & $0 \%$ \\
Nada importantes & 0 & $0 \%$ \\
TOTAL & 105 & $100 \%$
\end{tabular}

Tabla $N^{\circ} 11 .-$ ¿Qué tan importante considera que sus productos sean comercializados?

Proyectos para el plan de desarrollo integral en las comunidades de Palenque de la Provincia de Los Ríos

El plan contiene tres programas basados en las industrias del biocomercio, cada industria se desarrolló varios proyectos que son articulados con las estrategias planteada hasta el año 2030. A continuación, se detallan cada industria y proyectos que contiene el Plan de Desarrollo Integral.

\begin{tabular}{|c|c|c|}
\hline INDUSTRIAS & PROYECTOS & ESTRATEGIAS \\
\hline  & $\begin{array}{l}\text { Elaboración y venta de barras de } \\
\text { cacao amargo y semi amargo } \\
\text { basado en cultivo de cacao } \\
100 \% \text { orgánico. } \\
\text { Cultivo de uvilla y tomate cherry } \\
\text { y vegetales orgánicos. } \\
\text { Procesamiento en harina de maíz } \\
\text { 100\% orgánica. }\end{array}$ & $\begin{array}{l}\text { - Una agricultura y silvicultura sostenible. } \\
\text { - Producción } 100 \% \text { orgánica. } \\
\text { - Elaboración de abono orgánico. } \\
\text { - Pesca en rio sostenible para la alimentación loca. } \\
\text { - Mayor control en la prevención de pérdida de } \\
\text { biodiversidad en el mundo. } \\
\text { - Recuperación de los esteros naturales. } \\
\text { - Desarrollar actividades para la capacitación de } \\
\text { productos perdidos en las comunidades del }\end{array}$ \\
\hline
\end{tabular}




\begin{tabular}{|l|l|c|}
\hline & $\begin{array}{l}\text { Procesadora de pulpa de frutas } \\
\text { (papaya, guanábana mango, } \\
\text { ciruelas y maracuyá) }\end{array}$ & $\begin{array}{c}\text { Cantón Palenque. } \\
\bullet \text { Implementar tecnología para la producción de } \\
\text { abono orgánico. }\end{array}$ \\
\cline { 2 - 3 } & $\begin{array}{l}\text { Elaboración de queso fresco y } \\
\text { manjares. }\end{array}$ & \\
\hline
\end{tabular}

\begin{tabular}{|c|c|c|}
\hline INDUSTRIAS & PROYECTOS & ESTRATEGIAS \\
\hline \multirow{3}{*}{ 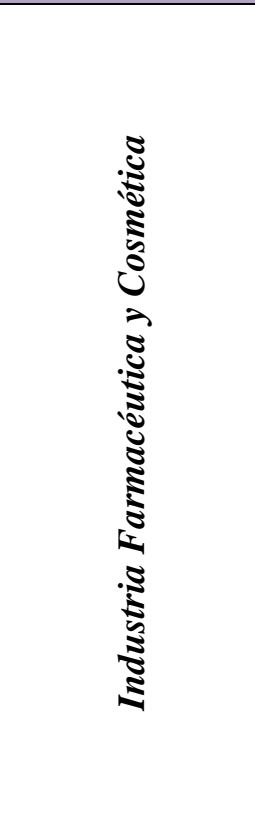 } & $\begin{array}{l}\text { Implementación de } \\
\text { huertos con planta } \\
\text { medicinales para la } \\
\text { elaboración de té } \\
\text { (llantén, menta, } \\
\text { orégano, hierba Luisa) }\end{array}$ & \multirow{3}{*}{$\begin{array}{l}\text { - Recuperación de plantas medicinales propia de la zona. } \\
\text { - Elaboración de tinte naturales para la industria cosmética. } \\
\text { - Implementación de maquillajes basado en el maíz y arroz } \\
\text { producido orgánicamente. } \\
\text { - Desarrollar programa de capacitación para la industria } \\
\text { farmacéutica. } \\
\text { - Articular capacitaciones con las Instituciones responsables } \\
\text { de los programas de salud. } \\
\text { - Recuperar los conocimientos ancestrales sobre medicina } \\
\text { natural. } \\
\text { - Recuperar la flora para fomentar la producción de miel. } \\
\text { - Transmitir a la nueva generación estos conocimientos de } \\
\text { medicina naturales }\end{array}$} \\
\hline & $\begin{array}{l}\text { Fabricación y venta de } \\
\text { maquillajes: polvo } \\
\text { compacto a base de } \\
\text { harina fina de maíz } \\
\text { orgánico. }\end{array}$ & \\
\hline & $\begin{array}{l}\text { Producción de Miel de } \\
\text { abeja. Como medicina } \\
\text { y endulzante natural. }\end{array}$ & \\
\hline
\end{tabular}

\begin{tabular}{|c|c|c|}
\hline INDUSTRIAS & PROYECTOS & ESTRATEGIAS \\
\hline 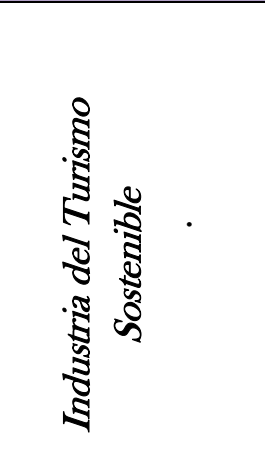 & $\begin{array}{l}\text { Turismo comunitario y } \\
\text { ecológico: paseos a } \\
\text { caballo, caminatas por } \\
\text { el lugar, paseos en } \\
\text { canoa o balsa en el } \\
\text { estero y hostería } \\
\text { comunitaria rustica } \\
\text { elaborada con madera } \\
\text { y caña }\end{array}$ & $\begin{array}{l}\text { - Controles estrictos sobre especies exóticas invasoras } \\
\text { - Mayor control en la prevención de la flora y fauna } \\
\text { - Tener accesibilidad a las atracciones y recursos naturales } \\
\text { centrada en el turismo ecológico. } \\
\text { - Fomentar a la población lo importante en innovar, las } \\
\text { cadenas de valor. } \\
\text { - Turismo ecológico como herramienta para mejorar la } \\
\text { economía de la zona. } \\
\text { - Articular los recursos económicos disponibles de forma }\end{array}$ \\
\hline
\end{tabular}






\section{Conclusiones.}

Los factores socioeconómicos que afectan al desarrollo del recinto Piscano están relacionados a las condiciones políticas, económicas de las autoridades de turno sean estas: locales, cantonales o nacionales; teniendo poco acceso a la educación, servicios básicos y muy poca información que permita impulsar el emprendimiento mediante el cooperativismo, se evidencia que los habitantes han deforestado gran parte de la tierra, dañando la biodiversidad del sector. A pesar de toda esta situación se pueden desarrollar proyectos que mejoren las condiciones de vida de los habitantes y recuperar la biodiversidad. Pero se necesita de la colaboración de empresa pública privada para fomentar y desarrollar las industrias del biocomercio y dar oportunidades a nuevos emprendimientos.

Existen varios proyectos sustentables que se pueden implementar aprovechando que la población económicamente activa que en su mayoría son jóvenes con deseo de mejorar sus condiciones de vida. Ellos conocen bien el sector, su cultura y costumbre que pueden convertirse en aliado para el desarrollo de estos proyectos mediante la transferencia de conocimiento ancestrales, aprovechando la flora, fauna y el turismo ecológico en el sector permitiendo desarrollar actividades agrícola, ganadera, pesca entre otras. 
Los gobiernos seccionales tienen la capacidad de impulsar y fomentar proyectos que mejoren las condiciones socioeconómicas de los habitantes, pueden implementar programas que impulsen el emprendimiento y dar una oportunidad a los pobladores para el desarrollo de su familia y por ende el desarrollo de los recitos, parroquias y todo el Cantón Palenque.

\section{Bibliografía.}

Guzman L, S., \& Gonzalez A, A. (2004). Biocomercio: una estrategia de desarrollo endógeno para risaralda. Scientia Et Technica, 5(25), 297-301.

Instituto de investigación de recursos biológicos Alexander Von Humboldt. (2000). Biocomercio: Estrategias para el desarrollo sostenible en Colombia. Bogotá: Instituto Humboldt.

Miller, G. (1990). Resource conservation and management. USA: Wadsworth Publishing Company.

Salvador, R., \& Gómez-Sal, A. (2003). Aprovechamiento sostenible de los pastizales. Ecosistemas, 12(3), 110 .

Scheffer, M., Carpenter, S., Foley, J., Folke, C., \& Walke, B. (2001). Catastrophic shifts in ecosystems. Nature, 413(1), 591-596. 